\title{
Infrared luminescence of annealed germanosilicate layers
}

\author{
M.S. Tokay ${ }^{a}$, E. Yasar ${ }^{\mathrm{a}, *}$, S. Ağan ${ }^{\mathrm{a}}$, A. Aydınlı ${ }^{\mathrm{b}}$ \\ a Department of Physics, Kirlkkale University, 71450 Kirlkkale, Turkey \\ ${ }^{\mathrm{b}}$ Department of Physics, Bilkent University, 06800 Ankara, Turkey
}

\section{A R T I C L E I N F O}

\section{Article history:}

Received 18 July 2013

Received in revised form

21 October 2013

Accepted 25 October 2013

Available online 8 November 2013

Keywords:

Infrared luminescence

Germanosilicate

Photoluminescence spectroscopy

Raman scattering

Annealing

\begin{abstract}
A B S T R A C T
In the light of growing importance of semiconductor nanocrystals for photonics, we report on the growth and characterization of annealed germanosilicate layers used for Ge nanocrystal formation. The films are grown using plasma enhanced chemical vapor deposition (PECVD) and post-annealed in nitrogen at temperatures between 600 and $1200{ }^{\circ} \mathrm{C}$ for as long as $2 \mathrm{~h}$. Transmission electron microscopy (TEM), Raman scattering and photoluminescence spectroscopy (PL) has been used to characterize the samples both structurally and optically. Formation of Ge precipitates in the germanosilicate layers have been observed using Raman spectroscopy for a variety of PECVD growth parameters, annealing temperatures and times. Ge-Ge mode at $\sim 300 \mathrm{~cm}^{-1}$ is clearly observed at temperatures as low as $700{ }^{\circ} \mathrm{C}$ for annealing durations for $45 \mathrm{~min}$. Raman results indicate that upon annealing for extended periods of time at temperatures above $900{ }^{\circ} \mathrm{C}$; nanocrystals of few tens of nanometers in diameter inside the oxide matrix and precipitation and interdiffusion of $\mathrm{Ge}$, forming SiGe alloy at the silicon and oxide interface take place. Low temperature PL spectroscopy has been used to observe luminescence from these samples in the vicinity of $1550 \mathrm{~nm}$, an important wavelength for telecommunications. Observed luminescence quenches at $140 \mathrm{~K}$. The photoluminescence data displays three peaks closely interrelated at approximately 1490 , 1530 and $1610 \mathrm{~nm}$. PL spectra persist even after removing the oxide layer indicating that the origin of the infrared luminescent centers are not related to the Ge nanocrystals in the oxide layer.
\end{abstract}

(c) 2013 Elsevier B.V. All rights reserved.

\section{Introduction}

There is currently great interest in nanometer sized $\mathrm{Si}$ and $\mathrm{Ge}$ structures following the observation of the efficient visible photoluminescence (PL) from porous Si [1], since this could open new possibilities for indirect gap semiconductors as new materials in optoelectronic applications. In particular, PL properties of $\mathrm{Si}$ nanocrystals ( $\mathrm{nc}-\mathrm{Si}$ ) have widely been studied and the relationship between the size of nc-Si and the PL peak energy has been revealed experimentally [2]. Many approaches to the realization of $\mathrm{Si}$ nanocrystals in a variety of matrices have been proposed. $\mathrm{Si}$ nanocrystals in insulating matrices, such as $\mathrm{SiO}_{2}$, are also considered candidates for future memory devices [3]. Intense work is under way to realize a $\mathrm{Si}$ laser [4]. Silicon nanocrystals in $\mathrm{SiO}_{2}$ typically form at relatively high temperatures, such as $1100{ }^{\circ} \mathrm{C}$, when annealed for $1 \mathrm{~h}$ or more and exhibit tunable photoluminescence due to size controlled nanocrystals formed by appropriate annealing conditions.

On the other hand, germanium (Ge) also is an indirect band gap semiconductor similar to silicon in many respects except for a smaller band gap. Ge containing $\mathrm{SiO}_{2}$ thin films can be obtained

\footnotetext{
*Corresponding author. Tel.: +90 31835742 42; fax: +90 3183572461 .

E-mail addresses: erdemyasar@kku.edu.tr, erdem.yasar@gmail.com (E. Yasar).
}

through, among many different techniques, ion implantation or plasma enhanced chemical vapor deposition (PECVD) of germanosilicate layers $[5,6]$ to name a few. However, Ge nanocrystals form at much lower annealing temperatures and durations as opposed to Si nanocrystals. While annealing temperatures of $800{ }^{\circ} \mathrm{C}$ and durations of a few minutes is typical to obtain Ge nanocrystals, Ge clusters of $2-3 \mathrm{~nm}$ sizes have been claimed to have formed even at annealing temperatures as low as $300{ }^{\circ} \mathrm{C}$ when annealed for $30 \mathrm{~min}$ [7]. However, lattice fringes of these nanocrystals have not been observed casting shadow on their crystallinity. Both TEM and Raman scattering have been employed to observe the formation of Ge nanocrystals in single and multilayers [8]. Extensive photoluminescence work yielded mixed results. Dutta [9] reported observing blue luminescence from Ge nanocrystals and claimed that PL is due to quantumconfined electronic transitions despite insufficient data. Paine et al. [10] have observed photoluminescence at $580 \mathrm{~nm}$ obtained from samples by $\mathrm{H}_{2}$ reduced $\mathrm{Si}_{0.6} \mathrm{Ge}_{0.4} \mathrm{O}_{2}$ and postannealed $750{ }^{\circ} \mathrm{C}$ which they attributed to Ge nanocrystals. Ge nanocrystals prepared by the sol-gel method in $\mathrm{SiO}_{2}$ and three photoluminescence peaks in the range of 2.0-2.3 eV were attributed to Ge nanocrystals [11]. Maeda [7] has studied Ge nanocrystals in $\mathrm{SiO}_{2}$ prepared by the cosputtering method and have observed both blue $(3.1 \mathrm{eV})$ and visible $(2.2 \mathrm{eV})$ photoluminescence and analyzed the data considering the quantum confinement model as well as Ge: E' luminescence centers in glasses 


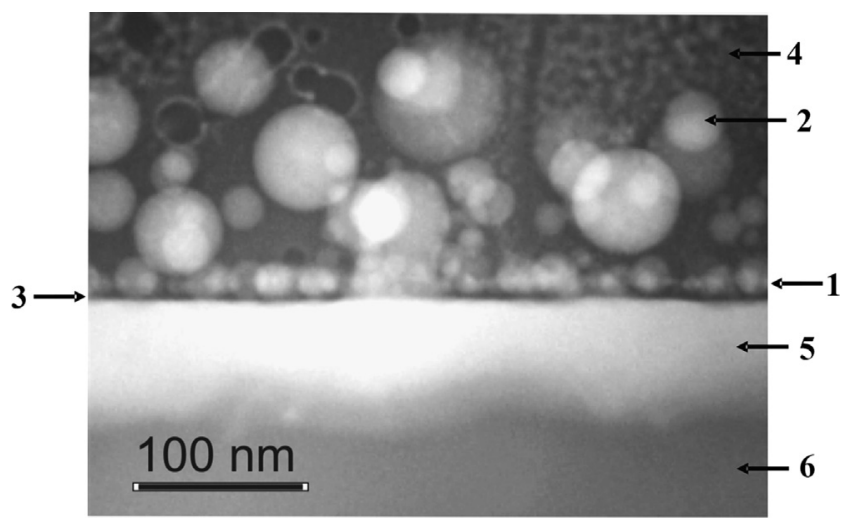

Fig. 1. Dark field STEM image of a sample annealed at $1000{ }^{\circ} \mathrm{C}$ for $1 \mathrm{~h}$. Ge nanocrystals are formed in the vicinity of the interface (number 1 and 2). Note the presence of two layers with two distinct average sizes of Ge nanocrystals. A nanocrystal free $\mathrm{SiO}_{2}$ interface oxide (number 3 ) and oxide close to the surface devoid of Ge nanocrystals, (number 4) is observed. Ge diffuses into Si substrate for an average thickness of $50 \mathrm{~nm}$ and $\mathrm{Si}$ substrate (number 5). Si substrate is also indicated (number 6).

and structural transitions of nanocrystal Ge, favoring the former model. Takeoka [12] has studied the near infrared photoluminescence in the range of $0.88-1.54 \mathrm{eV}$ from Ge nanocrystals prepared by the cosputtering method and concluded that the observed luminescence is due to radiative recombination of electron-hole pair confined in Ge nanocrystals. Torchynska et al. [13] have studied Ge nanocrystals in $\mathrm{SiO}_{2}$ and have concluded that all bands in the range of 1.6-2.35 eV are due to defects in $\mathrm{SiO}_{x}$ whereas PL bands in the range of $0.75-0.85 \mathrm{eV}$ are attributed to excitonic recombination inside Ge nanocrystals. It is thus clear from the literature that origin of photoluminescence from Gedoped silicate layers is still not clear. Much work has been devoted to study the electrical properties of Ge nanocrystals in $\mathrm{SiO}_{2}$ matrices [14]. Charging and discharging of Ge nanocrystals have been studied for flash memory applications. The possibility of charge storage in quantized levels of Ge nanocrystals has been shown [15].

In this work, $\mathrm{Ge}$ nanocrystals in $\mathrm{SiO}_{x}$ matrix were prepared by plasma enhanced chemical vapor deposition of $\mathrm{SiO}_{x}$ doped with Ge followed by postannealing of these layers. Both short term anneals as well as prolonged annealing has been carried out in nitrogen environment in the range of temperatures from 600 to $1200{ }^{\circ} \mathrm{C}$. Both the formation of Ge nanocrystals in the oxide matrix as well as diffusion and intermixing of Ge with $\mathrm{Si}$ in the substrate and the formation of SiGe alloy have been observed by TEM and Raman spectroscopy. Photoluminescence in the visible as well as in the near infrared is studied both at low and room temperatures. Photoluminescence in the near infrared is studied in detail because of the important optical communication wavelength region of $1.3-1.5 \mu \mathrm{m}$. Persistence of the photoluminescence even after the removal of the oxide layer containing the Ge nanocrystals suggests that, Ge islands on the Si substrate and SiGe alloy that forms at the interface of the oxide layer with the Si substrate, should also be considered for the origin of the observed luminescence.

\section{Experimental procedure}

The $\mathrm{SiO}_{x}$ :Ge films were grown in a PECVD reactor (PlasmaLab $8510 \mathrm{C}$ ) on $\mathrm{Si}$ substrates using $185 \mathrm{sccm} \mathrm{SiH}_{4}\left(2 \%\right.$ in $\left.\mathrm{N}_{2}\right), 45 \mathrm{sccm}$ $\mathrm{NH}_{3}$ and $120 \mathrm{sccm}$ flow rate of $\mathrm{GeH}_{4}$ (2\% in $\mathrm{He}$ ) as precursor gases, at a substrate temperature of $350{ }^{\circ} \mathrm{C}$, a process pressure of 1000 mTorr under an applied RF power of $10 \mathrm{~W}$. The samples were then annealed under nitrogen environment in a quartz oven at temperatures ranging from 600 to $1200{ }^{\circ} \mathrm{C}$ as long as $2 \mathrm{~h}$. Raman scattering experiments were carried out using a $1-\mathrm{m}$ double monochromator with GaAs photomultiplier and photon counting electronics. Various lines of an $\mathrm{Ar}$ ion $\left(\mathrm{Ar}^{+}\right)$laser and a $35 \mathrm{~mW}$ $\mathrm{He}-\mathrm{Ne}$ laser at $632.8 \mathrm{~nm}$ were used to excite the samples. Photoluminescence spectroscopy in the infrared is carried out at low temperatures with a $50 \mathrm{~cm}$ single pass monochromator equipped with a large area InGaAs detector. A closed cycle refrigerator is used down to $15 \mathrm{~K}$.

Cross section of the samples was observed with a transmission electron microscopy (TEM). The samples for the TEM observations were prepared by standard procedures in cross-section orientation and view edge on. Mechanical and $\mathrm{Ar}^{+}$thinning techniques were used to thin down the samples. $\mathrm{Ar}^{+}$at $5 \mathrm{keV}$ incident at $9-12^{\circ}$ was used. To minimize $\mathrm{Ar}^{+}$damage, the accelerating voltage was lowered down to $1 \mathrm{keV}$ in the final stages of the thinning process. The structural characterization was carried out with a JEOL 2010F field-emission transmission electron microscope operated at $200 \mathrm{keV}$.

\section{Results and discussion}

Fig. 1 shows a crosssectional dark field STEM image for a typical PECVDgrown $\mathrm{SiO}_{x}$ : Ge films annealed at $1000{ }^{\circ} \mathrm{C}$ for $1 \mathrm{~h}$. Upon annealing, crystallization of $\mathrm{Ge}$ is observed in the samples. TEM image shows that these nanocrystals fall into two groups. These two groups are composed of small nanocrystals with an average size of $15 \mathrm{~nm}$ and large nanocrystals that have an average size of $50 \mathrm{~nm}$. From the TEM micrography, a 3-5 nm thick layer of oxide on the Si substrate is observed to be free of Ge nanocrystals (number 1). Furthermore, $\mathrm{Ge}$ is observed at the $\mathrm{Si} / \mathrm{SiO}_{x}$ interface mixed with Si forming SiGe alloy. Thin layers or islands of Ge may also be present at the interface. It is suggested that Ge nanocrystals from $\mathrm{GeO}_{2}$ form due to an exchange reaction with Si diffusing in from the substrate into the oxide layer forming $\mathrm{SiO}_{2}$ and leaving elemental Ge behind [9]. The fact that Ge nanocrystals form only in the vicinity of the $\mathrm{Si}$ substrate seems to corroborate this mechanism. EDAX analysis of the substrate close to the $\mathrm{Si} / \mathrm{SiO}_{x}$ interface as well as the narrow band of contrast with the $\mathrm{Si}$ substrate at the interface seen in the TEM images suggest the presence of Ge on and in the Si substrate. All this is indicative of diffusion of Ge through the oxide layer and the formation of the SiGe layer at the silicon substrate-oxide interface.

Fig. 2 displays the results of Raman measurements from the same samples displaying the evolution of Ge nanocrystal formation upon annealing at temperatures in the range of $600-1200{ }^{\circ} \mathrm{C}$. As an example, we show the spectra for samples in the annealing temperature ranges of $600-1200{ }^{\circ} \mathrm{C}$ for $45 \mathrm{~min}$. The spectrum remains virtually unchanged for the annealing temperatures less than $600^{\circ} \mathrm{C}$. We observe a very broad $\left(\sim 40 \mathrm{~cm}^{-1}\right)$ asymmetric peak centered around $291 \mathrm{~cm}^{-1}$ indicative of the quasiamorphous nature of the $\mathrm{Ge}$ for samples annealed at $600{ }^{\circ} \mathrm{C}$ dominates the spectrum. We also note that the sharp rise on the right culminates in a very small peak at $299.27 \mathrm{~cm}^{-1}$ mixing into the quasiamorphous peak. Presence for this peak suggests that $600{ }^{\circ} \mathrm{C}$ is the onset of Ge crystallization as observed by Raman spectroscopy. Si substrate is observed at $520.4 \mathrm{~cm}^{-1}$. If the annealing temperature is raised to $700{ }^{\circ} \mathrm{C}$, a sharp peak at $299 \mathrm{~cm}^{-1}$, now $10 \mathrm{~cm}^{-1}$ in width, (not shown) is accompanied by a wide shoulder on the low frequency side. The sharp peak is a clear sign of Ge nanocrystal formation accompanied by a range of smaller Ge nanostructures. We note that this peak is at a lower frequency than the Ge mode in bulk Ge. This is most likely due to phonon confinement in small crystals. This peak becomes stronger at $299.8 \mathrm{~cm}^{-1}$ and narrower $\left(5.3 \mathrm{~cm}^{-1}\right)$ and the broad quasi-amorphous structure disappears 


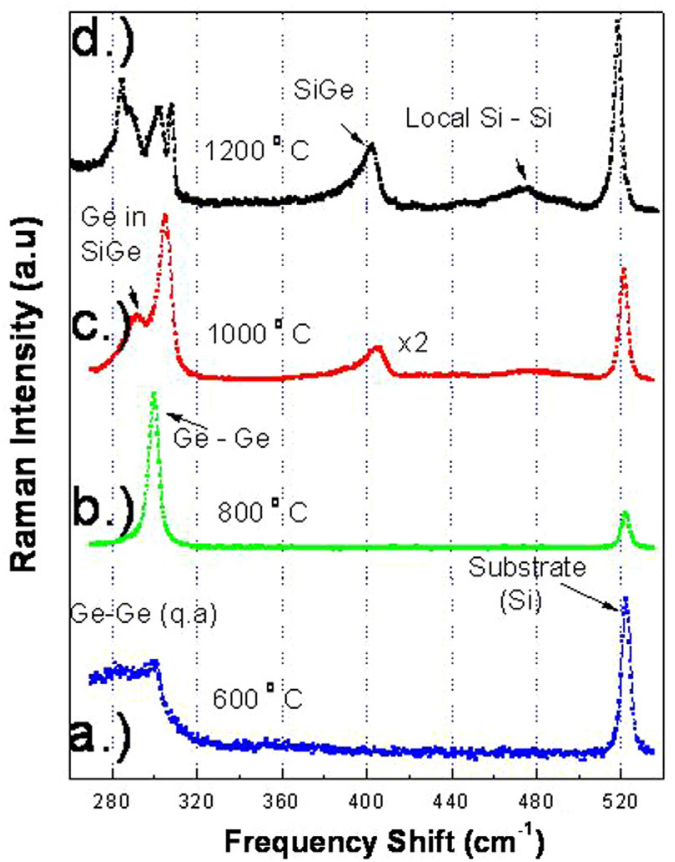

Fig. 2. Displays the Raman spectra of PECVD grown $\mathrm{SiO}_{x}$ :Ge films displaying the evolution of Ge nanocrystal formation upon annealing at temperatures in the range of $600-1200{ }^{\circ} \mathrm{C}$ from 40 to $120 \mathrm{~min}$.

as the annealing temperature is increased up to $800{ }^{\circ} \mathrm{C}$, Fig. $2 \mathrm{~b}$. At $900{ }^{\circ} \mathrm{C}$ (not shown) the Ge-Ge mode displays a double peak structure. These are located at 300.5 and $306.5 \mathrm{~cm}^{-1}$. The peak at lower frequency is attributed to Ge-Ge modes of the SiGe alloy at the interface while the higher frequency component of this double peak is due to the Ge nanocrystals in the oxide layer. This assignment has been confirmed with oxide removal experiments. After the removal of the oxide only the lower frequency component remains. In addition to the features associated with Ge nanocrystals, the spectrum now displays a weak but clearly discernible asymmetric peak centered at $410 \mathrm{~cm}^{-1}$ indicating the formation of SiGe alloy at the oxide Si substrate interface. In fact, if the oxide layer is completely removed with dilute HF solution, the same broad peak at $410 \mathrm{~cm}^{-1}$ is still observable. Furthermore, a very weak and broad peak centered at $482 \mathrm{~cm}^{-1}$ accompanies the SiGe at $410 \mathrm{~cm}^{-1}$. This peak is attributed to local $\mathrm{Si}-\mathrm{Si}$ modes and is expected with the formation of SiGe alloy. Finally, the Si substrate peak observed as a sharp peak centered about $520.5 \mathrm{~cm}^{-1}$.

Raman data for samples annealed at $1000{ }^{\circ} \mathrm{C}$ is shown in Fig. 2c. Two peaks associated with Ge modes down shift to 293.5 and $305.5 \mathrm{~cm}^{-1}$. The asymetric peak also down shift to $405 \mathrm{~cm}^{-1}$ and is now clearly pronounced. The Si-Si local mode is still quite broad but slightly down shifted to $476 \mathrm{~cm}^{-1}$. Si-Si mode due to the substrate remains at $521 \mathrm{~cm}^{-1}$. Sample was also annealed at $1200{ }^{\circ} \mathrm{C}$. The $\mathrm{Ge}-\mathrm{Ge}$ double peaks now evolve into three peaks located approximately at 308,302 and $285 \mathrm{~cm}^{-1}$. We speculate that the broad third peak at the low frequency side of the spectrum is due to SiGe alloys of varying compositions. The SiGe peak is now located at $403 \mathrm{~cm}^{-1}$ and the $\mathrm{Si}-\mathrm{Si}$ local mode remains at approximately the same position $\left(474 \mathrm{~cm}^{-1}\right)$ as that in the spectrum of $1000{ }^{\circ} \mathrm{C}$. Notably the $\mathrm{Si}$ substrate peak is also down shifted to $518.6 \mathrm{~cm}^{-1}$ suggesting that it is under stress.

Photoluminescence spectroscopy on these samples revealed very little in the visible part of the spectrum. Typically, a peak centered around $550 \mathrm{~nm}$ is observed and known to be due to defect states in the glassy matrix. The spectra in the near IR on the other hand have a broad peak center around 1550 nm, Fig. 3. The

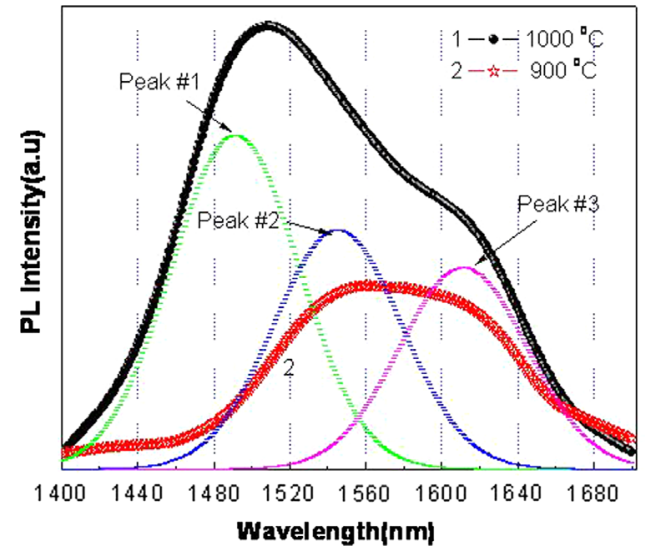

Fig. 3. Low temperature ( $15 \mathrm{~K}$ ) IR PL spectra of $\mathrm{SiO}_{x}$ :Ge films annealed at 900 and $1000{ }^{\circ} \mathrm{C}$ for $45 \mathrm{~min}$

spectra in Fig. 3 is from two samples annealed at 1000 and $900{ }^{\circ} \mathrm{C}$ for $45 \mathrm{~min}$ and consists of three peaks centered $\sim 1490(0.832 \mathrm{eV})$, $\sim 1530(0.810 \mathrm{eV})$ and $\sim 1610(0.770 \mathrm{eV}) \mathrm{nm}$. The effect of annealing temperature on the infrared spectrum may be better understood if we study samples annealed for different durations. Low temperature $(15 \mathrm{~K})$ IR spectra from such a sample annealed at $950{ }^{\circ} \mathrm{C}$ for 40,60 and $120 \mathrm{~min}$ is shown in Fig. 4. We again find a broad peak with well-defined peaks in the spectrum at $\sim 1516$ $(0.817 \mathrm{eV}), \sim 1524(0.813 \mathrm{eV})$ and $\sim 1533(0.808 \mathrm{eV}) \mathrm{nm}$, a clear blue shift of the spectra with increasing annealing duration. The data can be deconvoluted well with three Gaussian peaks.

The temperature dependence of the photoluminescence has also been studied, Fig. 5. We find that the highest intensity is obtained at the lowest temperature and as the temperature of the sample is raised during the measurement the peak intensity decreases. The signal to noise ratio detoriates as the temperature reaches $120 \mathrm{~K}$ and any sign of a photoluminescence signal cannot be distinguished beyond $140 \mathrm{~K}$.

Several possible mechanisms may be considered to explain the data. Among these are luminescence from dislocations in the SiGe alloy, luminescence from $\mathrm{Ge}$ or $\mathrm{SiGe}$ islands in or on the $\mathrm{Si}$ substrate and $\mathrm{Ge}$ nanocrystals in the $\mathrm{SiO}_{x}$ matrix. To test the latter consideration, the oxide layer has been removed in a dilute $\mathrm{HF}$ solution and the photoluminescence experiment was repeated. The observed spectrum is almost identical with those obtained when the oxide layer was in fact.

Photoluminescence from Ge and SiGe islands on Si has been studied by numerous authors. Kamenev et al. [16] has studied photoluminescence from nanometer sized clusters with Ge core and SiGe shell grown on Si by molecular beam epitaxy under near Stranski-Krastanov growth mode conditions and found a broad band covered the range from 0.85 to $0.95 \mathrm{eV}$ which broadens and shifts down to the range $0.65-0.90 \mathrm{eV}$ as the Ge concentration increases. Talalaev et al. [17] have studied $\mathrm{Ge} / \mathrm{Si}$ multilayer structures with Ge quantum dots. The observed photoluminescence spectra cover a broad range between $0.75-0.90 \mathrm{eV}$. Eberl et al. [18] measured photoluminescence characteristics of selfassembled SiGe nanostructures and observed a broad peak between 0.75 and $0.90 \mathrm{eV}$.

We have also studied the variation of the emission $\mathrm{A}$ band maximum intensity versus the excitation laser intensity. Excluding the saturation region at the highest intensities, the experimental data can be fitted by the simple power law of the form $I \alpha L^{\gamma}$ where $I$ is the PL intensity, $L$ is the excitation laser intensity and $\gamma$ is a dimensionless exponent. It was found that the PL intensity at the emission band maximum increases sublinearly with respect to the excitation laser intensity Fig. 6. It is well known that for an 


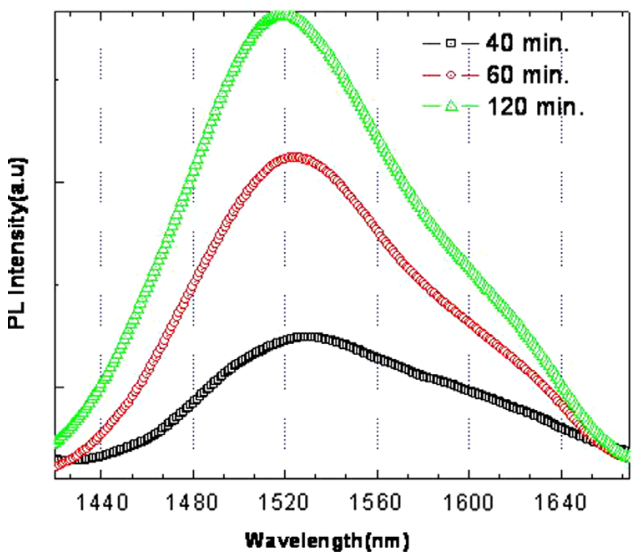

Fig. 4. Low temperature ( $15 \mathrm{~K}$ ) IR PL spectra of $\mathrm{SiO}_{x}$ :Ge films annealed at $950{ }^{\circ} \mathrm{C}$ with 40, 60 and 120 min annealing times.

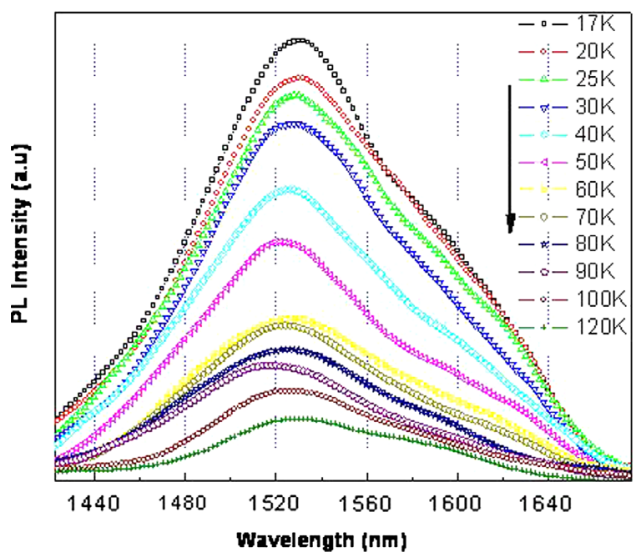

Fig. 5. Temperature dependent IR PL spectra of $\mathrm{SiO}_{x}$ : Ge films annealed at $1200{ }^{\circ} \mathrm{C}$ for $1 \mathrm{~h}$ under $632.8 \mathrm{~nm}$ excitation.

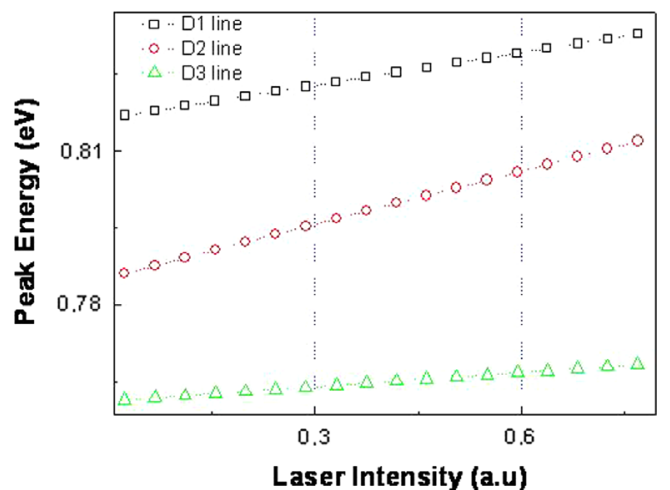

Fig. 6. Excitation intensity dependent low temperature IR PL of samples annealed at $1200{ }^{\circ} \mathrm{C}$ during $1 \mathrm{~h}$

excitation laser photon with an energy exceeding the bandgap energy $E_{g}$, the coefficient $\gamma$ is generally $1<\gamma<2$ for the free and bound-exciton emission, and $\gamma \leq 1$ for free-to-bound and donoracceptor pair recombinations [19].

Studies of the PL temperature dependence show that at high temperatures, the PL intensity drops exponentially, and the activation energies of PL thermal quenching are shown in Fig. 7 of $\mathrm{SiO}_{x}$ : Ge films annealed at $1200{ }^{\circ} \mathrm{C}$ during $1 \mathrm{~h}$. The transitiontemperature of $140-160 \mathrm{~K}$ can be understood as that the carriers transport is hopping from site to site when $\mathrm{T}<140 \mathrm{~K}$, so there is

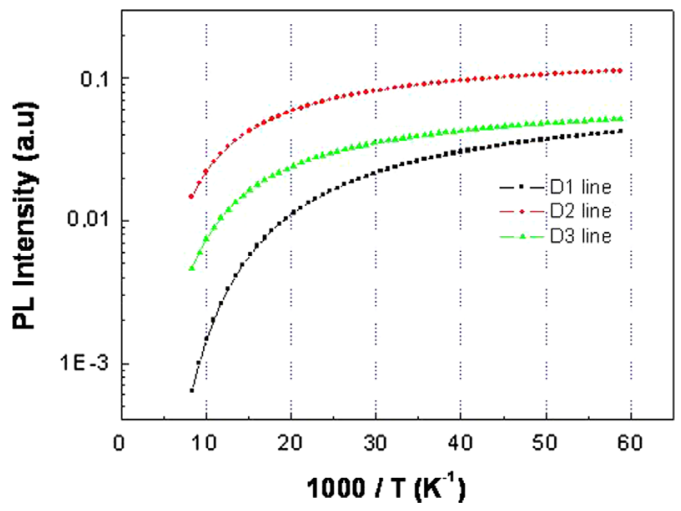

Fig. 7. Arrhenius plot of $\mathrm{SiO}_{x}$ : Ge films annealed at $1200{ }^{\circ} \mathrm{C}$ during $1 \mathrm{~h}$. Temperature dependence of PL intensity at the A band of sum. The arrow shows the starting point of the intensive quenching.

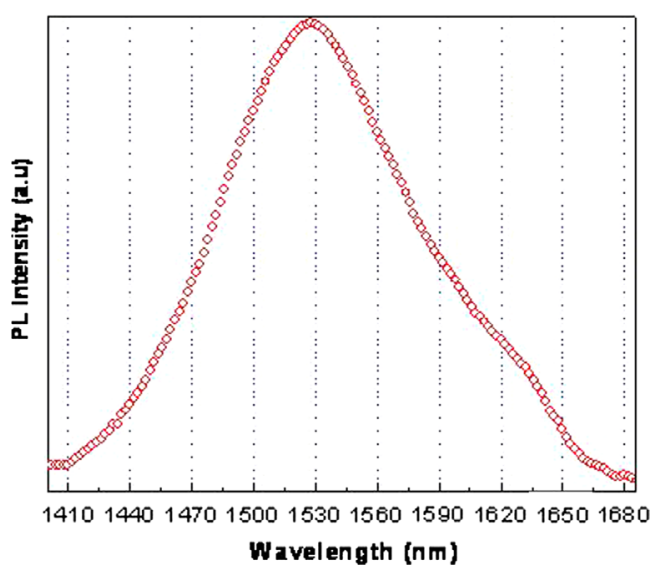

Fig. 8. PL results come from nanocrystals via removing oxide layer for the sample annealed at $1000{ }^{\circ} \mathrm{C}$ at $45 \mathrm{~min}$.

not much chance for them to be captured by the nonradiative center; when temperature is increased to $\mathrm{T}>160 \mathrm{~K}$, the carriers are thermally emitted to the band edge and then are easily captured by the non-radiative recombination centers. Fig. 7 shows the temperature dependence of the A band maximum intensity as a function of the reciprocal temperature in the $11.5-81 \mathrm{~K}$ range. $A$ rapid thermal quenching of the $A$ band is observed above $T=35 \mathrm{~K}$. The experimental data for the temperature dependence of the PL intensity at the emission band maximum (I) can be fitted by the following expression:

$I(T)=I_{0} \exp \left(\frac{\Delta E}{k T}\right)$

where $I_{0}$ is a proportionality constant, $\Delta E$ is the thermal activation energy and $k$ the Boltzmann constant. The semilogarithmic plot of the emission band intensity as a function of the reciprocal temperature gives a straight line in the $35-81 \mathrm{~K}$ region. In conclusion, we have shown that the photoluminescence of $\mathrm{Ge}$ self-assembled quantum nanocrystals is strongly dependent on the power excitation density.

Finally, we have examined to the PL results come from nanocrystals via removing oxide layer from the sample annealed at $1000{ }^{\circ} \mathrm{C}$ at $45 \mathrm{~min}$ shown in Fig. 8 . At $15 \mathrm{~K}$ maximum peak was obtained $1528 \mathrm{~nm}$. There is a red shift with comparing unremoved oxide layer sample annealed at the same temperature and time.

There are several possibilities for the source of the observed IR $\mathrm{PL}$ form high temperature annealed $\mathrm{Ge}$ doped $\mathrm{SiO}_{x}$ films on $\mathrm{Si}$ substrates. First, Si substrate itself is known to have defect related 
PL emission in this part of the spectrum. These well documented emission lines dubbed as D1 through D4 occur 1528, 1423, 1317 and $1238 \mathrm{~nm}$ [20]. The physical origin of these emission lines have been studied both theoretically [21] and experimentally. Using density functional theory, it has been shown that clusters of $\mathrm{Si}$ with defect states may be responsible for the emitted PL. Furthermore such defectrelated lines are also observed in SiGe alloys [22]. In partially, relaxed samples some of the D lines are not observed. In totally relaxed samples all four D lines are observed with varying degrees of intensity. Secondly, PL emission from bandtoband recombination of SiGe alloys is also a possibility. PL emission from SiGe structures are observed [23] in this part of the spectra as it lies between the bandgap of Si and that of Ge. Nearband gap luminescence from SiGe alloys have been studied [24]. Low temperature spectra shows excitonic structures shifting from red to blue as the Ge content of the SiGe alloy decreases. Ge-Ge, $\mathrm{Si}-\mathrm{Ge}$ and $\mathrm{Si}-\mathrm{Si}$ modes of the system has been characterized as a function of SiGe composition and does not show any dispersion making it difficult to use the Raman line positions as calibration tools for the determination of composition of our films. Molecular beam epitaxy of SiGe and Ge on Si has also been attempted [25]. Ge islands growing on Si substrates show a blue shifted PL emission $\sim 1500 \mathrm{~nm}$ for very low coverage. The data is explained in terms of quantum confined Ge clusters, which show PL red shifting upon increasing cluster size. Finally, interdiffusion of Ge into Si has been studied with concominant PL emission in the IR in the form of D lines [26]. As our Raman data shows the presence of SiGe in our samples our PL data seems to be consistent with defectrelated PL emission from SiGe during the interdiffusion of $\mathrm{Ge}$ into Si. Alternatively, PL emission from SiGe alloys is also a possibility. The data is explained in terms of quantum confined $\mathrm{Ge}$ clusters, which show PL red shifting upon increasing cluster size.

These localized luminescence centers likely originate from defect centers at the $\mathrm{Ge} / \mathrm{Si}$ interface or defect centers inside the Ge clusters. We increased the Ge concentration in the $\mathrm{SiO}_{x}$ films. The exclusive presence of Peak 1 and Peak 2 emission bands in the samples containing amorphous $\mathrm{Ge}$ nanoclusters indicates that these amorphous clusters must play a critical role in the PL process. A possible explanation of the PL is that excitons are generated in the Ge nanoclusters or in bulk $\mathrm{Si}$ and then decay at defect centers that are located either within the nanocrystals or at the $\mathrm{Si}$ interface. The presence of Ge nanoclusters produces high energy excitons, higher than the bulk Ge band gap energy and a high density of interface states. This model has been used extensively to explain the luminescence properties from $\mathrm{Si}$ and $\mathrm{Ge}$ nanocluster systems [27]. No visible luminescence was observed from the samples, however, a broad photoluminescence peak around $1500 \mathrm{~nm}$ was observed at low temperatures in samples with germanium precipitates.

It is necessary to understand the enhanced PL properties of the Ge nanocrystals for possible further enhancement. It is known that the surface and/or interface states of the crystals have a great effect on their PL properties. These surface/interface states can act as either radiative or nonradiative recombination centers, consequently leading to a significant enhancement of PL intensity. Oxygen-deficient centers also can act as deep traps. Therefore, one can expect further PL enhancement if the crystal size is further decreased, the oxidation condition is optimized and/or the nonradiative defect density is decreased [28].

This broad peak $(0.8-1.0 \mu \mathrm{m})$ is probably due to interfacial oxygen-deficient defects between the oxide and the ncGe.

Theoretically, quantum dot infrared photodetectors have been predicted to have high gain and low dark currents compared to quantum wire infrared photodetectors. It has also been reported that the PL intensity increases drastically as the size decreases. In general, the red and near-infrared PL previously observed are considered to originate from the recombination of electron-hole pairs between the widened band gap of nc-Si (quantum size effects).

\section{Conclusions}

In this study, nanostructures of Ge formed by precipitation of germanium in PECVD grown germanosilicate films were studied using TEM, Raman and PL spectroscopies. We have fabricated $\mathrm{SiO}_{x}$ : Ge thin films using PECVD. Cross-sectional TEM images show the formation of nanocrystal structures in $\mathrm{SiO}_{x}$ matrix and Raman scattering was used to monitor the formation of Ge nanocrystals for as-grown and nitrogen and vacuum annealed samples. Annealing results in the formation of SiGe alloy at the oxide Si interface. Photoluminescence spectra obtained from the $\mathrm{SiO}_{x}$ : Ge suggests that luminescence originates from defect centers and is not consistent with the quantum confinement based luminescence expected from Ge nanocrystals despite the fact that Raman scattering clearly points to their presence.

Annealing of $\mathrm{SiO}_{x}$ :Ge films result in of formation of $\mathrm{Ge}$ nanocrystals and SiGe alloy the oxide Si interface; the formation of two distinct types of structures has been verified through Raman scattering measurements and TEM micrographs.

Low temperature photoluminescence in the IR around $1500 \mathrm{~nm}$ has been identified as Ge islands formed at the silicon and oxide interface, an important wavelength for telecommunications. Formation of such structures by simple PECVD growth and annealing is interesting because of their potential use as infrared emitters.

\section{Acknowledgment}

We thank to Aykutlu Dana for sample growth. This work is supported by TÜBITAK (Turkish Scientific and Technical Research Council) through contact 109T129.

\section{References}

[1] J. Pandiarajan, N. Jeyakumaran, N. Prithivikumaran, Adv. Mater. Res. 584 (2012) 290.

[2] J. Alberto, L. López, J. Carrillo López, D.E. Vázquez Valerdi, G. García Salgado, T. Díaz-Becerril, A. Ponce Pedraza, F.J. Flores Gracia, Nanoscale Res. Lett. 7 (2012) 604.

[3] C. Bonafos, M. Carrada, G. Ben Assayag, S. Schamm-Chardon, J. Groenen, A. Slaoui, P. Dimitrakis, P. Normand, Mater. Sci. Semicond. Process. 15 (2012) 615.

[4] Ding Li, Yong-Bin Chen, Ming Lu, Mater. Lett. 89 (2012) 9.

[5] N.L. Rowell, D.J. Lockwood, A. Karmous, P.D. Szkutnik, I. Berbezier, A. Ronda, Superlattices Microstruct. 44 (2008) 305.

[6] R. Xu, W. Li, J. He, Y. Sun, Ya-Dong Jiang, J. Non-Cryst. Solids 365 (2013) 37.

[7] Y. Maeda, Phys. Rev. B 51 (3) (1995) 1658.

[8] S. Agan, A. Dana, A. Aydinli, J. Phys-Condens. Matter 18 (2006) 5037.

[9] A.K. Dutta, Appl. Phys. Lett. 68 (9) (1996) 1189.

[10] D.C. Paine, C. Caragianis, T.Y. Kim, T. Shigesato, T. Ishahara, Appl. Phys. Lett. 62 (22) (1996) 2842

[11] M. Nogami, Y. Abe, Appl. Phys. Lett. 65 (20) (1994) 2545.

[12] S. Takeoka, M. Fujii, S. Hayashi, Phys. Rev. B. 58 (12) (1998) 7921.

[13] T.V. Torchynska, A.V. Hernandez, Y. Goldstein, J. Jedrzejewskii, S. Jiménez Sandoval, J. Non-Cryst. Solids 352 (2006) 1152.

[14] H. Liu, W. Winkenwerder, Y. Liu, D. Ferrer, D. Shahrjerdi, S.K. Stanley, J.G. Ekerdt, S.K. Banerjee, IEEE Trans. Electron. Dev. 55 (12) (2008) 3610.

[15] A. Dana, I. Akça, O. Ergun, A. Aydınlı, Physica E 38 (1-2) (2007) 94.

[16] B.V. Kamenev, J.M. Baribeau, D.J. Lockwood, L. Tsybeskov, Physica E 26 (1-4) (2005) 174

[17] V.G. Talalaev, G.E. Cirlin, A.A. Tonkikh, N.D. Zakharov, P. Werner, U. Gösele, J.W. Tomm, T. Elsaesser, Nanoscale Res. Lett. 1 (2006) 137.

[18] K. Eberl, O.G. Schmit, R. Duschl, O. Kienzle, E. Ernst, Y. Rau, Thin Solid Films $369(1-2)(2000) 33$.

[19] W. Wang, K. Wang, D. Han, Bed Poudel, Xiaowei Wang, D.Z. Wang, Baoqing Zeng, Z.F. Ren, Nanotechnology 18 (7) (2007) 5707.

[20] V. Higgs, F. Chin, X. Wang, J. Mosalski, R. Beanland, J. Phys. Condens. Matter 12 (2000) 10105. 
[21] A.T. Blumenau, R. Jones, S. Öberg, P.R. Briddon, T. Frauenheim, Phys. Rev. Lett. 87 (2001) 1874041

[22] E.A. Steinman, V.I. Vdovin, T.G. Yugova, Semicond. Sci. Technol. 14 (1999) 582

[23] K. Terashima, T. Ikarashi, D. Tweeti, K. Miyanaga, T. Tatsumi, M. Tajima, Appl. Phys. Lett. 65 (1994) 601

[24] J. Weber, M. Alonso, Phys. Rev. B 40 (1989) 5683.
[25] J.P. Noël, N.L. Rowell, D.C. Houghton, D.D. Perovic, Appl. Phys. Lett. 61 (1992)

[26] K. Shum, P.M. Mooney, J.O. Chu, Appl. Phys. Lett. 71 (1997) 1074.

[27] A.A. Shklyaev, M. Ichikawa, Appl. Phys. Lett. 80 (2002) 1432.

[28] W.Z. Wang, B. Poudel, J.Y. Huang, D.Z. Wang, S. Kunwar, Z.F. Ren, Nanotechnology 16 (2005) 1126. 